\title{
The Degrees of Understanding and the Inferential Component of Understanding
}

\author{
Stefan Petkov*
}

Received: 22 August 2019 / Revised: 11 October 2020 / Accepted: 28 September 2020

Abstract: Current debates on the nature of explanatory understanding have converged on the idea that at least one of the core components of understanding is inferential. Philosophers have characterized the inferential dimension of understanding as consisting of several related cognitive abilities to grasp a given explanation and the nexus of complementing explanations to which it belongs. Whilst analyses of both the subjective epistemic abilities related to grasping and objective features of the inferential links within explanations have received much attention, both within theories of explanation and in the literature on understanding, the criteria for evaluating the specific structure and organization of explanatory clusters or nexuses has received much less attention. Nevertheless, two notable exceptions stand out-Khalifa's characterization of an explanatory nexus, and theories of explanatory unification. I take Khalifa's ideas, together with the basic criteria of successful explanatory unification, as my starting point. To both I make some corrections and additions, in order to arrive at a more robust notion of an explanatory nexus and ultimately show that its structural properties and the inter-explanatory relations it contains are relevant to the resulting understanding.

* Beijing Normal University

(D) https://orcid.org/0000-0001-9152-574X

- School of Philosophy, Beijing Normal University, Room A801, Front Main building, Beijing Normal University, No. 19 Xinjiekouwai Str, Haidian District, Beijing, China

$\bowtie$ yaggdrasil@yahoo.com

() The Author. Journal compilation (C) The Editorial Board, Organon F.

This article is distributed under the terms of the Creative Commons Attribution-NonCommercial 4.0 International Public License (CC BY-NC 4.0). 
I propose to represent such nexuses as directed graph trees and show that some of their properties can be related to the degree of understanding that such nested explanatory structures can offer. I will further illustrate these ideas by a case study on an ecological theory of predation.

Keywords: Ecological theory of predation; explanatory understanding; scientific explanations; unification.

\section{Introduction}

The problem of explanatory understanding has received significant attention in the recent literature of epistemology and philosophy of science (see Baumberger et al. 2017 for an overview). The exact definition of understanding $^{1}$ is a matter of an ongoing dispute, but most analyses have converged on the idea that at least one of the key differences between mere knowledge of a correct explanation of a phenomenon and understanding the phenomenon has an inferential character (Newman 2014; Grimm 2010; Khalifa 2017; De Regt 2015). The inferential character of the explanatory understanding of a given fact, or a factual domain, has been analysed in the literature in two complementary ways, related to both the inferential properties of singular explanations and the organization of inter-linked explanations:

1. As a subjective ability to evaluate the quality of the available explanations (Grimm 2010; Newman 2014; Khalifa 2017), the ability to grasp the explanations (e.g. arriving at new inferences based on the original explanatory blueprint) and to relate these explanations together forming an explanatory nexus;

2. As the objective features of explanatory inferences in terms of the type of relation between the explanans and the explanandum (Kostić

1 For stylistic purposes, here I will use "understanding" and "explanatory understanding" interchangeably to refer to the same epistemic good of comprehending a phenomenon by an available explanation. A further clarification on this notion can be found in section 2 . 
2018), the informativeness and relevance of the explanatory premises, and the quality of the so-called cluster or nexus of complementing explanations (Khalifa 2017).

If we take into account the idea that understanding is not a binary notion, but comes in degrees (Khalifa 2017; Newman 2014), we can then see that the degree of understanding of some fact or factual domain depends on both subjectively grasping the conceptual inferential relations within explanations or between complementing explanations of that fact or domain of facts, but also on objective features of these explanations and established inter-explanatory relations. Whilst analyses of the subjective epistemic abilities related to grasping, and the specific features of the inferential links within explanations, have received extensive attention, both in theories of explanation (Hempel 1965; Lewis 2000; Woodward 2003; Lange 2016) and in the literature on understanding (Baumberger et al. 2017), criteria for evaluating the specific structure and organization of explanatory clusters or nexuses has received much less attention.

Two notable exceptions stand out. In the literature on understanding Khalifa has introduced the general notion of an explanatory nexus (Khalifa 2017). He has suggested that the degree of understanding that an explanatory nexus provides is determined by comparative principles of completeness and likeness to scientific knowledge. However, his characterization of explanatory nexuses and the criteria for their evaluation have remained too broadly defined. ${ }^{2}$ In theories of explanations, unificationist theories have also conveyed the idea that explanations should not be considered in isolation. These theories have developed the idea that understanding should be judged both on the basis of the number of facts an explanation can cover (Kitcher 1989) and on the number of other explanations that an explanation can unify (Friedman 1974; Bartenbloth 2002). Therefore, unification can potentially provide the grounds for a more robust criterion for the evaluation of a cluster of explanations, in terms of their organization and their inter-explanatory relations.

2 Khalifa's analysis of completeness of grasp for instance is overly broad and as he himself notes is not susceptible to quantitative assessment (see Khalifa 2017, 10), for more details see section 2.3 . 
Here, I will take both Khalifa's idea of an explanatory nexus and the basic criteria of successful explanatory unification as my starting point. In both cases, I will make some corrections and build upon the existing views in order to define the notion of an explanatory nexus more robustly and to offer more precise criteria for its evaluation. My central effort will be to show that the organizational and structural properties of these nexuses also play an important role in determining the degree of understanding.

To give an outline of the paper. In the second section, I will present a short review of the literature on the subjective and objective features of inferential explanatory understanding. In the third section, I will link the core ideas of these analyses with explanatory unification. In the fourth section, I will lay the groundwork for a possible formal explication of an explanatory nexus as a directed graph tree. Making some corrections to both Khalifa's notion of a nexus and the basic criteria of unification, I will provide robust criteria of the degree of understanding that such nexuses can provide in terms of both the quantitative evaluation of subjective completeness of grasp and the evaluation of the objective properties of explanatory structures. I will also give a short illustration of these ideas by a case study on an ecological theory of predation.

\subsection{The subjective side of the inferential component of understanding}

The philosophical analysis of understanding has shown that the difference between mere knowledge of the correct explanation of a given phenomenon and understanding that phenomenon has a distinct subjective dimension. Typically, this dimension has been analysed under the concept of grasping. Grimm (2010) has described grasping as the ability "to work upwards" (so to speak) from a particular explanation towards the relevant fragment of a background theory, by mastering the general conceptual relations, or the dependence conditions, that are exemplified in the explanation.

An extension of the analysis of the subjective epistemic requirements of understanding has been made by Newman (2014). For him, understanding involves several distinct but related cognitive abilities such as semantic 
ability, comprehension ability and problem-solving ability. A test for these cognitive abilities would be to check the subject's ability to link the newly introduced explanatory inferences to pre-existing explanatorily relevant information, in order to solve novel problems.

To illustrate, reaching understanding of the phenomenon of flight, by learning the Bernoulli principle, as a result of answering the explanationseeking question "Why can the plane fly?", is not equivalent to merely knowing that the Bernoulli principle provides relevant information about the conditions which make flight possible. Understanding flight via this explanation means also being able to work out how the variables in the equation will co-vary in different circumstances. "Grasping" this explanation, for instance, will mean being able to infer that the theoretic maximum altitude of a given model of glider depends on the shape of its wings and the density of the air at different altitudes. Understanding in this sense involves the ability to analyse the explanatory relation between the explanans and the explanandum in terms of the conceptual relations exemplified in the explanation and thus arriving at the explanatory blueprint which the explanation exemplifies.

As Newman has shown, grasping will also involve linking the resulting explanatory blueprint to other available explanatory information for the purpose of solving novel problems. For example, linking the newly introduced Bernoulli principle to a background knowledge of thermodynamics will allow us to understand not only flight but also how injectors in steam locomotives work.

Even though our consideration of the idea of grasping clearly shows that explanations should not be considered in isolation, an exclusive focus on the subjective epistemic abilities that make possible the linking of newly introduced explanatory information to pre-existing explanatory knowledge will leave the analysis of understanding one-sided. Obviously, an objective evaluation of both the structural properties of explanatory inferences themselves and of how these inferences are organized together with other background explanatory information (producing larger and more encompassing inferential networks) is also essential.

However, as we shall see, the literature on theories of explanations and on explanatory understanding has focused for the most part only on 
analysing the relation between explanations and their explananda and less on providing criteria for the organization of such inferential networks.

\subsection{The objective side of the inferential component of understanding and singular explanations}

Most $^{3}$ of the classical literature on scientific explanations has been focused on analysing understanding in terms of the features of the inferential relation between the explanandum and the explanans. This strategy has been endorsed by most well-known theories of scientific explanation, such as the deductive-nomological model (Hempel 1965), and various causal approaches (Dowe 2000; Salmon 1998; Woodward 2003). The deductive-nomological model has been deployed to investigate this relation as a form of logical consecution of the lawful premise in the explanans that subordinates the explanandum; in causal theories of explanation the relation is described as counterfactual dependence (Lewis 2000) or difference making (Woodward 2003) between the cause (explanans) and the effect (explanandum). The introduction of distinctively mathematical explanations has further enriched this picture by also describing some scientific explanations in terms of the relation of logical constraint that a mathematical explanans imposes upon an ontic fact (explanandum) that is satisfied by it (Lange 2016).

Recently, Kostić (2018) has proposed a general criterion for assessing explanatory understanding based on the relation between the explanans and the explanandum. His central claim is that, because the goal of explanation is understanding, understanding is intimately linked with explanation. For him, this intimacy depends on the level of complexity in the structure of explanation in such a way that the more immediate the relation between the explanans and explanandum, the more immediate the understanding.

As a corollary, he also claims that. The explanation will provide more understanding and depth if the explanatory relation is more immediate. ${ }^{4}$

3 With the notable exception on theories of explanatory unification. See section 3.

4 Kostic has suggested that the most immediate relation between explanans and its explanandum is non-inferential in character and any type of explanatory inferences such as deductive-nomological explanations are necessarily more complex. 
These results from the fact that the immediacy also has as a by-product the possibility to apply that explanation to a large number of phenomena.

He defends these ideas by comparing minimal structure explanations (a type of distinctively mathematical explanations) with deductive-nomological or causal mechanistic explanations. Kostić shows that minimal structure explanations require simply grasping that the explananda satisfy a given mathematical structure and, as such, that the derived properties of that mathematical structure will also be exemplified by the explananda. If these properties are the ones which fall under the spotlight of explanatory seeking problems, then an explanation need only show that the explanandum instantiates the mathematical structure. As a corollary, he suggests that, due to the higher abstractness of these explanations, the explaining mathematical structure can be used to cover a large variety of facts, also offering significant explanatory depth. In contrast to this type of explanation, causal explanations, for instance, have a narrower scope, because they exemplify specific causal interactions and, as such, require specific ontic information which is not generalizable.

Here, I shall agree with the first element of his account - the more intimate the relation between the explanans and the explanandum the more immediate the understanding. The second claim, which links the explanatory depth of minimal structure explanations to the immediacy of the explanatory relation is more contestable and does not fit well with the account of unification I am going to develop here.

Firstly, it is possible that a minimal structure explanation satisfies the norm of intimacy between an explanans and explanandum but remains applicable to a very few phenomena. This is because the number of phenomena that exemplify a given mathematical structure is a contingent fact, which does not depend on the properties of the exemplified mathematical structure. After all, nothing in the immediacy of the relation between a mathematical explanans and a factual explanandum hints that such a relation should be satisfied by more than a single fact. Therefore, it is a contingent

Since the problem I address here is that of inter-explanatory relations and structures, I will leave open the problem of whether there are cases of non-inferential explanations. 
ontic issue whether a given mathematical structure, despite its abstractness, is applicable to a large number of facts or not.

Nevertheless, the inverse is certainly correct. The more general the explanation the less specific ontic information it contains and, therefore, the more immediate the relation between its explanans and explanandum. As such, more abstract or general explanations should stand higher in a possible hierarchy of interlinked explanations. As for determining the explanatory depth that such explanations can provide, and use it as a cubit for understanding, I shall not follow Kostić directly. Instead, I will try to develop a more complex view of unification that requires that the general applicability of a given explanation and the explanatory relation it exemplifies should always be taken into account in relation to other more general or more specific explanations. ${ }^{5}$

To conclude, if we take Kostić's account narrowly, as a criterion for evaluating singular explanations by determining the type of relation that holds between the explanans and the explanandum, and by comparing it with different explanatory strategies, it strikes me as fundamentally correct in its basic assumption of intimacy. Therefore, in what follows, I shall take it as a basic criterion for ordering explanations. The more generally applicable the explanation, the more intimacy it will display between its explanans and its possible explananda, and the higher it should stand in a hierarchy of interlinked explanations.

5 Broadly applicable explanations (as Kostić himself notes) naturally offer less particular information due to their abstractness. As such they are sometimes supplemented by specific causal mechanistic explanations. The ensuing deeper understanding can then be seen in terms not of broader applicability alone, but as the possibility of linking these more abstract explanations with more narrow causal explanations which offer specific ontic information. Moreover, following Woodward (2003), an account of unification cannot consider that phenomena that are describable by the same mathematical structure are unified e.g. it is possible that the same mathematical equation is used to describe several unrelated phenomena, however we cannot treat these phenomena as well unified under this mathematical equation. For more on the type of unification, I am going to develop see section 3.1. 


\subsection{Khalifa's combined criterion and explanatory nexuses}

In his book, Khalifa (2017) has developed a general analysis of scientific understanding. He has combined both the objective and subjective dimension of understanding by giving the following comparative criterion upon which the degree of understanding of some fact $p$ can be determined:

(EKS1) $S 1$ understands why $p$ better than S2 if and only if:
(A) Ceteris paribus, $S 1$ grasps $p$ 's explanatory nexus more com pletely than S2; or
(B) Ceteris paribus, S1's grasp of $p$ 's explanatory nexus bears greater resemblance to scientific knowledge than $S 2$ 's. (Kha- lifa 2017, 14)

As we can see from this definition, his criterion relies heavily on the notion of an explanatory nexus, which, for him, is the cluster of correct explanations of $p$, as well as the relations between those explanations. If we take the second criterion "the resemblance to scientific knowledge" as satisfied - e.g., all the explanations satisfy the accepted objective criteria for the inferential relation between explanans and explanandum - this leaves us with the second element, completeness. However, perhaps due to the general project of his book, Khalifa has left the notion of completeness too broadly defined. Under the heading of completeness, Khalifa has listed both the number of correct explanations and inter-explanatory relations grasped and the quality of these explanations and inter-explanatory relations. Moreover, he has described completeness of nexuses in a non-restricted way - as all the available explanations of a given fact. Even if explanations meet the criteria for scientific likeness (e.g., they are the best our science can offer) these explanations can hardly be organized in any robust way. Theories use many different explanatory strategies, including descriptions of broad mathematical dependencies, deductions derived from lawful generalizations, inferences based on phenomenological modelling; they may include approximations and idealizations, and so on. Without any criteria of the relations holding between these different explanatory products, the notion of a nexus remains (though intuitively appealing) very vague. Consequently, the notion of completeness, as Khalifa defines it, only enables a comparison of the 
relative degrees of understanding of different epistemic subjects in simple cases where one of the subjects lacks an explanation that the other possesses (Khalifa 2017, 10).

However, as we shall see, inter-explanatory relations between explanations can be defined more clearly and, given this, both the organization and the completeness of such nexuses can be more precisely described. If this is the case, not only the number of correct explanations, but also their organization and the contrast class of phenomena that they consider, will all be relevant to the resulting understanding.

Khalifa's definition provides a strong starting point. However, in order to render the problem of evaluating explanatory nexuses more manageable, I will narrow my focus to explanatory nexuses limited to a single theory. I will also assume that the examined explanatory structures contain only genuine scientific explanations of one particular type (e.g. that these explanations are all lawful deductions, or describe causal dependences, or are subject to some distinctively mathematical constraints such that all are properly "science-like").

Two problems then stand out. Firstly, how are we to organize explanatory nexuses; and secondly, by which criteria are we to judge the interexplanatory relations that such nexuses display?

To make an analogy with the classical theory of explanations: if that theory's central problem has been the characterization of the appropriate types of explanans/explanandum relations, then similarly, for the theory of understanding based on grasping explanatory nexuses, an analysis of explanatory structures grasped in terms of their inter-explanatory relations and organization must also be made.

\subsection{Explanatory unification as a starting point for evaluating explanatory nexuses}

So far, we have seen that the degree of understanding of some fact or factual domain will depend not only the subjective grasp and objective evaluation of the inferential relations within the available explanations, but also on establishing and evaluating inter-explanatory relations. The problem 
then is to present a more precise notion of such structures of explanations, and show how these structures can be evaluated based on their organizational inter-explanatory properties. I believe this idea can be developed on the basis of the existing analyses of explanatory unification. We can start building up a suitable notion of unification by following in chronological order the key accounts of Friedman (1974), Kitcher (1989) and Bartelborth (2002).

The theory of explanatory unification was pioneered by Friedman in 1974. In his original paper, he asked the same question as that raised in the contemporary topic of understanding: in virtue of what do scientific explanations provide understanding? His answer was that scientific theories generate understanding by offering two types of explanations: explanations which reduce the number of independent phenomena by subsuming them under general laws ${ }^{6}$, and more general explanatory inferences that subsume the independent laws. Even though Friedman's account suffered from some technical problems ${ }^{7}$, his central idea of a hierarchy of explanations remains valuable. Roughly, we can paraphrase it as linking the degree of understanding that a cluster of explanations offers to how unified this cluster is. Kitcher (1989), however, did not build directly on this idea of a hierarchy, or interlinked structure of explanations; instead, he focused on describing successful unification in terms of a theory that manages to subsume its domain under the smallest possible set of explanations. To explicate this idea, he developed the concept of an explanatory pattern. Such patterns for Kitcher serve as skeletal blueprints of particular deductive explanatory arguments. ${ }^{8}$ For example, a general pattern for evolutionary dynamics can be:

SNS:

Explanans: $x$ and $y$ are members of $n$, differentiated by a variant on an inherited trait $z ; x$ and $y$ are competing in $E$, where $x$ is fitter than $y \rightarrow$ Explanandum: $x$ 's size is larger than $y$ in $E$ in some $n^{\prime}>n$ ).

6 Friedman worked under the tenets of the deductive-nomological model.

7 It accepted simple conjunctions of independent laws as explanations of these laws (Kitcher 1976).

8 Famously, Kitcher has defended a position of deductive chauvinism - all explanations are ultimately reducable to causal explanations. I shall not follow him in this idea. Instead, I will assume explanatory pluralism. 
Here $\mathrm{x}$ and $\mathrm{y}$ are the demographic sizes of sub-populations of the same species, differentiated by some trait $\mathrm{z}, \mathrm{E}$ is the relevant environmental factors and $\mathrm{n}$ designates the generation number. A simple theory of evolution contains only this pattern plus a set of filling instructions for the dummy letters. When these filling instructions are exhausted all the instances of that pattern can be generated and the domain of the theory can be closed. It is clear that SNS is highly systematic, because it covers every possible evolutionary scenario, excluding only population states resulting from genetic drift. The downside is that such a general pattern offers very little information and thus understanding of actual evolutionary factors. Therefore, systematization or generality alone are insufficient as a measure of understanding. To amend this issue, Kitcher provided another requirement for successful unification. A unified theory for him is such a structure of explanations that manages not only to systematize its domain under a small number of explanatory patterns but also one in which these patterns are as stringent as possible (e.g. they provide as much particular information as possible).

Jointly satisfying the two requirements of stringency and systematization has been shown to be a serious problem for Kitcher's account, because they clearly pull in different directions (Morrison 2000). Beside the tension between stringency and systematization, there is also the question (left open by Kitcher) of how organizations that display different numbers of patterns and varying degrees of systematization and stringency can be compared (Kitcher 1989, 435).

Barelbroth (2002) has essayed a resolution of these issues by extending Friedman's original notion of a hierarchy of explanations. He makes use of the formal apparatus of structuralism to propose that unification is successful in generating understanding only if it manages to embed the explananda into a hierarchy of explanatory models each of which displays a different level of generality. His and subsequent accounts of unification (Petkov 2015) describe this hierarchy as a treelike structure, at the root of which lie the most general explanatory patterns. These most abstract patterns present the core concepts of the theory, their relations, and how they can be applied in order to map the factual domain (Petkov 2015). These explanatory patterns are then progressively extended by specifying the core conceptual links in such a way that more and more specific explanations can be constructed. 
Optimally, this should result in a series of progressively more stringent explanations that should exhaust in a contrastive manner the factual domain and also offer deeper understanding.

Such a picture of theories as hierarchies of linked explanations that exhaust a particular domain is suggestive of the notion of an explanatory nexus. However, we cannot directly deploy the criteria of stringency and systematization in the evaluation and description of explanatory nexuses. This is because, firstly, Khalifa has defined explanatory nexuses as collections of the explanations of singular facts, whilst both Grimm's grasping and unification are geared instead to describing explanations and organizations of explanations in terms of their broader applicability to more than a singular explanandum. Recall that both grasping and unification are evaluated in terms of the possibility of using a particular explanation of some fact as an exemplar of an explanatory blueprint that is then applicable to further phenomena similar to the explanandum. Moreover, as we saw, Khalifa defined explanatory nexuses in a non-restricted way - as all the available scientific explanations of the fact under scrutiny. This permits the inclusion of any number and any type of explanations, and, such clusters clearly does not meet the criteria for unified knowledge.

Another serious problem that this time emerges from the side of unification is the so called "ad explanandum" problem (Halnonen and Hintikka 1999; Petkov 2015). Halonen and Hintikka (1999) have criticized explanatory unification because it typically presents the embedding of more specific explanations into more general ones, as a type of inferential derivation. Halonen and Hintikka have suggested that this derivation implies that the explananda are considered as deductively closed or static. Whilst in fact particular explanations often include, specific ad explanandum information. This information is case sensitive and similarly to auxiliary conditions of deductive nomological explantions, is not generalizable nor it can be derived directly from the available explanatory information from the higher level explanations. Similarly, the notion of a general explanatory blueprint that is applied serially (e.g. grasped), cannot actually work, because at each application of that general explanatory pattern we would need to supply additional knowledge specific to the particular explananda, which is then effectively equivalent to discovering new explanations. 
Both of these issues present a significant challenge to the project of quantitatively comparing explanatory nexuses in terms of grasping and to the development of a qualitative account of such nexuses in terms of their unification.

\subsection{An interrogative account of explanatory nexuses limited to a particular domain}

Notwithstanding the above, I believe that, given certain corrections to both our idea of unification and Khalifa's notion of a nexus, we shall see that we can render these concepts much more robust, and also provide some guidelines for a quantitative analysis of the degree of understanding that a nexus provides, in terms of its content and structural properties.

We can start with the curious historiographical observation about the literature of unification. Both its proponents and critics seem to have been preoccupied with Kitcher's notion of an explanatory pattern and the problem of how such patterns hang together. However, in his original essay, Kitcher developed the concept of explanatory patterns, along with the requirements for stringency and systematization, as an extension of van Fraassen's pragmatic approach to explanations. Therefore, in order to meet the challenges of explicating explanatory nexuses more clearly, I will, so to speak, "go back to the roots".

I take Kitcher's basic idea that a systematization of a domain is in fact achieved by patterns which are recoverable by van Fraassen's account. I will use this idea as a starting point and link it with the notion of explanatory hierarchies of Barelbroth and subsequent accounts of unification. As we shall see, this will lead to a resolution of the ad explanandum problem raised by Halonen and Hintikka. It will also help us reach a notion of unification suitable for the evaluation of explanatory nexuses.

Bas van Fraassen's (1980) pragmatic approach to explanations starts with the idea that explanatory questions do not arise in a vacuum, devoid of background information. Instead, they are reducible to contrastive questions of the type Q: "Why $\mathrm{x}$ and not $\mathrm{y}$ ?", where $\mathrm{x}$ is the factual explanandum and $y$ is some contrast class of other unrealized possibilities. Under this condition, an explanation takes the form of an answer A: "It is the case 
that $\mathrm{x}$ and not $\mathrm{y}$ because A" (for further details and formalization see van Fraassen 1980 and Kitcher 1989). In van Fraassen's original formulation the answer must (ideally) be a direct answer. A direct answer is one that reveals explanatorily relevant information, which, by its introduction, shows the topic of the queston $x$ to be the only factually true statement out of the contrast class $y$.

Two elements are essential in such an approach to explanations. Firstly, relevance of the explanatory answer to the question and, secondly, the construction of the appropriate contrast class.

A typical example will be a causal explanation in which we have the question "Why did the window shatter when it got hit by the ball, as opposed to the ball just bouncing off it?", here the answer can contain, say, relevant information about the force of the throw, the weight of the ball, and the tensile strength of the glass. As such, the answer provides case specific conditions (or ad explanandum information) that show why the shattering of the window was the only realized possibility (for similar contextual approach to causation see Schaffer 2005; Reiss 2013a, 2013b).

If we look at this example closely, we will see this to be a case of a very specific question, which receives an equally specific answer; as such it can be treated as an instance of a very stringent pattern. However, under unification we are not typically asking why a singular fact has occurred given any range of possibilities. Instead, we are asking why the range of possibilities is as it is, but also why this fact has occurred under this specific range of possibilities. We are first trying to determine the correct contrast class and then to determine why $x$ has occurred within that class.

In other words, we are not dealing with a singular explanatory seeking question and a singular answer. Instead, we are dealing with a generalization which defines the borders of the contrast class, and then with a series of explanatory seeking questions, nested into each other, that aim to exhaust this contrast class or, more specifically, to zero in on the fact under scrutiny. This nesting works by introducing information that leads to providing a more and more narrow contrast class, until we zero in on the specific singular explanandum. If we take this idea into account, we can easily arrive at the notion of a hierarchy, and also treat this hierarchy as effectively describing an explanatory nexus. 
Instead of following Kitcher's approach and defining unified theories statically, we can include more and more factually specific information in our answers. This will permit us to resolve the ad explanandum problem, raised by Halonen and Hintikka. In fact, it will also help us to arrive at the notion of an explanatory nexus and its organization. Similarly, instead of describing nexuses statically as all the available explanations for some facts, we can define clusters of explanations as a series of nested explanatory questions and answers, along with the contrast classes of phenomena which they introduce.

Based on this we can determine the completeness of an explanatory nexus not as all the available explanations for $x$, but as a sort of recursive interrogative game that includes variations of $x$ and some distinct (but still theoretically relevant) facts $y$ :

Q1: Why $x \ldots x_{n}$ along with some $y \ldots y_{n}$ can occur?

A1: $x \ldots x_{n}$ and $y \ldots y_{n}$ can occur because A1.

Q2: given A1 why $x \ldots x_{n}$ occurs and not $y$ ?

A2: $x \ldots x_{n}$ occurs and not $y$ because A1.

Q3: given A1, A2 why $x_{k}$ occurs and not $x_{n}$ ?

A3: $x_{k}$ occurs and not $x_{n}$ because A3.

For example, we would like to understand why Julius Caesar died. We can start with the question: Was Caesar killed or did he die of natural causes? Given the answer that Caesar was killed, we can ask further: Was he poisoned, stabbed, cut, burned, drowned, suffocated or beaten with a blunt object? Once we establish that Caesar was stabbed we can try to answer further forensic questions about the stabbing: Were any vital organs punctured? Did he die of shock, or because of blood loss, or because of organ failure? Were the lungs or heart punctured?

Such a recursive game will establish a series of nested questions and answers relating more general questions to more particular ones. Two important points emerge. Firstly, in order to make the resulting structure manageable, we must not ask why-questions in any non-restricted way - we should not mix facts about the forensics of the Caesar's death with these of possible motives of his assassins, the political situation in Rome at the time, the psychological dispositions of Brutus etc. 
Secondly, even in this restricted fashion, this structure of nested explanations meets Halonen and Hintikka's problem, because at each step we are introducing new information via more specific and fine-grained answers, which in turn permits us to appreciate more and more minute differences between our contrast class of phenomena. Therefore, better understanding intuitively results from grasping not only the basic contrastive difference between some theoretically relevant phenomena $\mathrm{x}$ and $\mathrm{y}$, but also more and more minute variations of $\mathrm{x}$.

An interesting problem emerges here. Van Fraassen's original account treated contrast classes unrestrictedly. As a consequence, we can use any contrast class of non-realized possibilities as a basis of our explanation. For instance, our problem of Cesare's death becomes the muddled: Did Cesar ever died or was he abducted by extra-terrestrial time travelers from Mars, who also hypnotized Brutus and other members of the senate, leaving only a dummy dead clone of Cesar on his original place? Therefore, an important problem is how to restrict the contrast class, so we can arrive at a more manageable and realistic structure

As a solution, firstly, we should consider a contrast class that is limited to the actual domain of our explaining theory. Secondly, we should include only genuine scientific explanations, such that the relation between the topic of the question (explanandum) and the answer (explanans) satisfies the requirement of some of the accepted models of explanations. For instance, if we have a structure containing only causal explanations and counterfactual answers, we are not normally including any possible causal influences or all and any variations of the explanandum (See Woodward 2003 and his notion of serious possibilities). Moreover, we can also deploy Kostić's criterion of intimacy between explanandum and explanans as a guideline on how to organize the explanatory structure- e.g., starting with explanations (explanans) with a more immediate relation to the explanandum, and moving towards more complex cases.

Taking these considerations into account we can also quantitatively determine-How unified the nexus of $x$ can be by solving the problem:

Prob 1: What is the least number of why questions that need to be answered in order to uniquely pick up $x$ from as rich as possible an initial contrast class? 
This problem introduces the requirement for stringency (as informative as possible) and systematization (as few as possible as systematic as possible explanations). Similarly, to the requirement for unification, the smaller the initial contrast class, the easier it will be to arrive at a maximally stringent and exhaustive structure, and vice versa. However, we saw that "grasping" requires evaluating explanations not only in terms of their stringency but also in terms of their broader applicability (systematization).; A such, the larger the contrast class considered, the more explanatory information we would need in order to zero in on the specific explanandum.

Similarly, the optimal organization and completeness of an explanatory nexus can be determined by the problem:

Prob 2: What is the least number of why questions that need to be answered in order to pick up each unique member of as rich as possible an initial contrast class?

The central idea is that we are trying first to establish a starting generalization which is as systematic as possible for as large as possible a contrast class of phenomena, and then trying to exhaust this contrast class by the smallest possible number of additional questions that we need to answer in order to exhaust the relative differences between all the phenomena in the contrast class. Such nexuses can be as open or as limited as one requires. For instance, for a complete theory of evolution, we would need an initial generalization like SNS, and then extensions of specific explanations that can ultimately map onto the origin events and population states of all the species on Earth. Obviously, most actual theories and their explanations will have a much narrower scope and can be, for all practical purposes, complete (Newtonian optics or the study of some simple model organisms in biology are good examples).

With this in mind, we can also determine the subjective completeness of grasp and the different degrees of understanding that epistemic agent $\mathrm{S}$ will have for $x$ by the problem:

Prob 3: How large is the contrast class of which $x$ is a member that $S$ considers, and what is the number of questions that $S$ needs to ask and receive an answer to in order to pick up $x$ uniquely from the contrast class? 
Naturally, the less information the agent requires in order to discover why $x$ has occurred as oppose to a large number of variations of $x$, the better he understands $x$. Armed with these ideas, we can also represent the resulting structure in a more robust way — as a hierarchical graph tree. By examining its structural properties, I hope to arrive at more precise responses to these problems, and, as such, also at quantitative criteria for evaluating explanatory nexuses.

\subsection{Explanatory structures as directed graph trees}

A starting point from which to describe these explanatory structures is to represent them as directed graphs. The simple case will be a direct or simple explanation, where $\mathrm{A}$ is the explanans and $\mathrm{B}$ the explanandum:

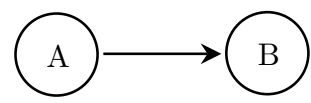

Fig. 1

In order to reach the notion of a pattern, and utilizing van Fraassen's pragmatics, we need only change the structure of explanatory seeking questions from "Why $\mathrm{x} 1$ and not $\mathrm{y}$ ?" to the exhaustive Q: "Why x1 xor x2?". Thus, $\mathrm{Q}$ along with two possible answers-A1 for $\mathrm{x} 1$ and A2 for $\mathrm{x} 2$ - can be represented as such:

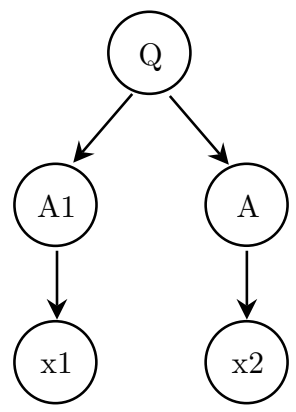

Fig. 2

Because an explanatory pattern is any structure in which the explanans applies to more than one explanandum, we can represent the case from fig 
2 as a pattern in which $\mathrm{A}$ encompasses both $\mathrm{x} 1$ and $\mathrm{x} 2$ and instantiates respectively two simple explanations $\mathrm{B}$ and $\mathrm{C}$. Recall that the difference between an explanation and a pattern is that a pattern instantiates more than one explanation. Therefore, this will also be the case for the most stringent pattern, which is just a step removed from a singular explanation. In order to represent exclusively the branching and the inter-explanatory relations of such a pattern, we can depict it as a simple binary tree:

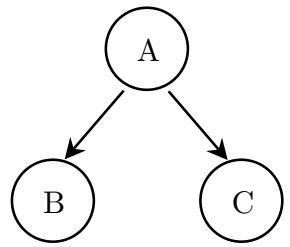

Fig 3.

The paths $\mathrm{A} \rightarrow \mathrm{B}$ and $\mathrm{A} \rightarrow \mathrm{C}$ should be read as simple explanations of facts, respectively, $\mathrm{x} 1$ and $\mathrm{x} 2$; and the structure as covering $A\{x 1, x 2\}$. Under this approach a root is always the most systematic node. The children are always more stringent and the leaves are always singular explanations that zero in on a particular explanandum. A pattern can cover any range $\operatorname{Rang}\{x \in N: 1<x\}$. Consequently, patterns can be ordered into levels according to their range and nested into each other, where, respectively, patterns of the type $A \mp B$ are cases in which $\mathrm{A}$ is a more stringent pattern for some of the $\mathrm{x}$ that the more systematic B covers. From this perspective, an optimal organization for the structure of such nested explanations will be a balanced binary tree (fig. 4):

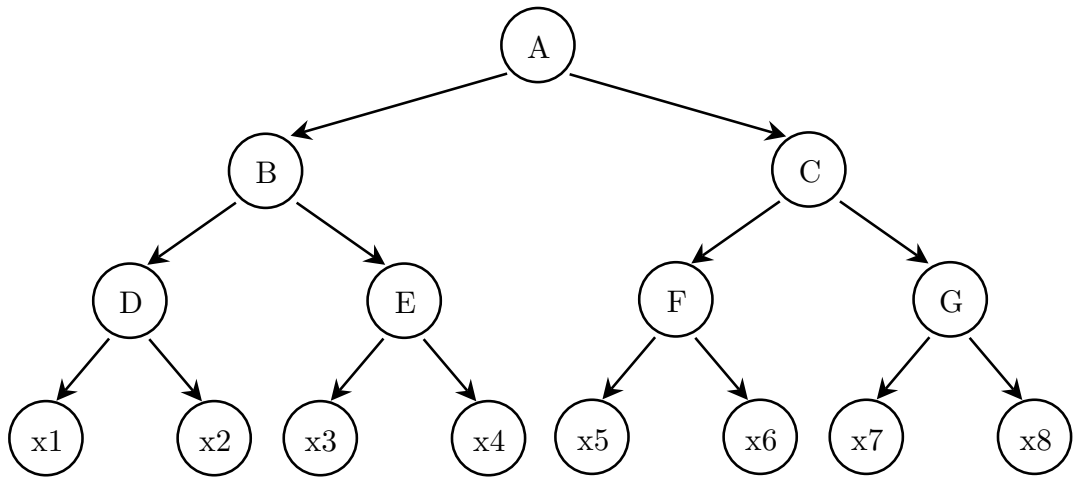

Fig. 4 
To be more precise, in such a tree there will be no leaves that directly connect with the root (with the exception of a structure with a single maximally stringent pattern that is not equivalent to a direct explanation - as in fig.3). All the leaves will be connected to a branch and all the branches including the root will have at most 2 children. Because at each level a branch will have at most 2 children, this will be a case of a structure that contains as stringent patterns as possible, that are completely systematic for the leaves. (Recall that the most stringent pattern has only 2 instantiations - see fig. 3).

Another property that we would desire is to keep the level $(l)$ of the structure as low as possible. The level of a node is defined by $1+$ the number of connections between the node and the root. For example, the root will have level 1, its two children 2, their four children 3 etc. (see fig. 4 for an example). Keeping the path from the root to each specific leaf as short as possible exemplifies the scenario in which we need to solve as few explanatory problems as possible in order to reach each domain specific fact. In other words, we are trying to obtain as stringent as possible and as systematic as possible structure. Therefore, a solution for Prob 2 (of optimal organization and completeness of an explanatory nexus) essentially reduces to a balancing problem for a binary tree.

The balance of a binary tree can be determined based on the relation between the level of the tree $l$ and the number of its leafs $N$, which is $2^{(l-2)}<$ $N \leq 2^{(l-1)}$. We can obtain a balanced structure when we have as few levels (l) as possible for $N$ number of leafs under the condition: $\log _{2} N+1 \leq l<$ $\log _{2} N+2 ; l=\left\lceil\left(\log _{2} N\right)+1\right\rceil$. It is also important to determine the number of nodes, because they represent our explanatory data. For a binary tree this is easy, since each nod can have at most 2 children. Therefore, the number of nods A can be determined based on the number of leafs $N$ as: $A=$ $2^{\left[\log _{2} N\right]}-1+N$.

With this in mind, the solution to Prob 1 of. how unified the nexus is for a balanced binary tree with root $R$ and $X\left(x_{i \ldots n}\right)$ number of leafs is simply $\forall x_{i \ldots n} \epsilon R=d x_{i \ldots n}$, where $d$ is the depth of a leaf $x$ (the number of edges we need to travel from the root to the leaf $\mathrm{x}$ ).

Finally, a solution for Prob 3-determining the subjective completeness of grasp will be found in the case of determining what subtree of the 
balanced tree $A$ an epistemic subject can recover, or how similar his explanatory nexus is to $A$. If his explanatory nexus is identical to $A$, then he completely grasps explanatory structure $A$. Determining the similarity between such graphs, cannot be only structural but must also contain evaluation of the explanatory data that each nod contains. Therefore, determining this similarity is a more complex issue that goes beyond the scope of the present text. However, some suggestions for comparative analyses of data graphs can be found in Zageret al (2008).

Obviously, any actual explanatory structures that we are going to deal with in the wild will fall short of this ideal scenario. They will display varying degrees of balance; they might be incomplete; they may have multiple roots; they may display varying heights in their explananda, and so on. Nevertheless, actual organizations of explanations can be examined as such directed graph trees and their degree of balance be measured. In the next section, I will suggest that, perhaps contrary to initial appearances, such structural analysis is not too idealized, and at least some fragments of theories that use simple nested mathematical models can be represented in this way.

Before moving on to the case study, a final note that I must make is that the approach to unification as a descriptive tool of explanatory nexuses or theoretic explanatory stores in general was inspired by the algorithm problems related with optimal organization of databases for solving problems in obtaining fast and effective searches. As such, the advances made by both computer science (Knuth, 1998) and formal analyses of such structures from descriptive set theory (Kechris, 1994), might offer interesting avenues of research.

\subsection{Case study - ecological models as explanatory structures}

To start building on the idea of a theory as a hierarchy of explanations, I will use an example from the ecological theory of predation. I believe, however, that similar analysis can be undertaken for any theory which has a clearly definable conceptual core and displays sufficient typicality in its explanatory strategies. 
Even though the complexity of ecological phenomena does not permit the same level of robust unification as displayed by law-based theories in physics, reconstructing the ecological theory of predation as a unified system is not a philosophical chimera. Some ecologists (Holt 2011; Ginzburg and Colyvan 2004, p.135) have already attempted such reconstructions and the present case study is based on their work.

My starting assumption is that the ecological theory of predation can be partially ordered as a hierarchy of models, where more specific models are nested into more general ones. The resulting hierarchical structure exhausts a significant fragment of the factual domain and its models are indispensable for explaining population dynamics. This structure of related models can also be examined as an organization of explanatory patterns. The resulting structural representation can provide valuable information on how ecological explanations offer understanding. But before proceeding onward, some preliminary clarifications must be made.

Firstly, proponents of the idea that scientific theories are collections of loosely related models might object that models are abstract or concrete objects and as such cannot be completely reduced to propositions, whilst the analysis developed here is exclusively concerned with explanations which are patently propositional. Without getting into the thorny problem of scientific representations, we can assume that models express beliefs about their targets, and that models are employed to make inferences about their targets. Consequently, models can be loosely investigated as sets of functions which interpret as parameters some of the core theoretic terms and predicates of the theory. Given an input that substitutes the parameters with values derived from observations, these functions will generate conclusion-like statements about observational targets. If the model manages to be representative of its targets, the conclusions made by the model, along with its fundamental assumptions, can be ordered in an explanatory inferential structure. ${ }^{9}$ Secondly, mixing models and explanatory patterns can result in a somewhat messy picture of theories. Nevertheless, I will try to

\footnotetext{
${ }^{9}$ This claim does not aim to diminish the significance of the philosophical theories of representation, nor to completely reduce the semantic view of theories to a syntactic one. My modest goal here is to establish that there is an intimate relation between explanatory patterns and formal models.
} 
present a clear picture as best as I can by giving examples of how explanatory patterns can be used to derive formal models and vice versa.

Any ecological theory starts with the assumption that the demographics of a population will depend on 3 factors: i.) $E$ environmental conditions (for instance available resources, fluctuations of the climate etc); ii.) $N$ factors specific for the organisms (as birth and death rates); iii.) $P$ factors due to interaction with other organisms (such as predation, competition for resources, parasitism, cooperation etc.). This very general framework of ecological relations can be specified as a root pattern:

GenEc:

Explanans: The influence of species-specific factors $N$ ', environmental factors $E$ and relations with other species $P \rightarrow$ Explanandum: determines the demographics of a population of organisms $N$ at a time $t$.

Based on it we can also build an initial mathematical expression (based on Holt 2011):

$$
\text { (eq. 1) } \frac{\mathrm{dN}}{\mathrm{dt}}=\mathrm{Nf}_{n}(\mathrm{~N}, \mathrm{P}, \mathrm{E})
$$

As in our toy case concerning natural selection, such a pattern, and the resulting model, are not very informative. GenEc, however, serves as a root pattern that draws the boundaries of the theory. It has only the systematizing function of drawing the boundaries of the domain of ecology. Such a pattern does not provide any contrast between the domain-specific facts. Consequently, it can only provide understanding if it is extended to more informative explanatory patterns that specify its core concepts (E, P and $\mathrm{N})$. A step in that direction is to specify only one of the conditions and examine the resulting contrast that such a pattern can provide.

The most straightforward way to extend one of the factors is to assume a function of birth-rate for $\mathrm{N}$ and construct a model of population growth:

$$
\text { (eq.2) } \frac{1}{N} \frac{d N}{d t}=r_{N}
$$

The solution of this simple function is exponential growth: $N(t)=$ $N_{0} \exp \left(r_{n} t\right)$. To represent it as a pattern we can interpret the function as receiving input information about a population of organisms at time $t^{0}$ and 
generating output populations states for $t^{\mathrm{n}}$. The model then gives rise to the pattern:

$\operatorname{Exp} G$ :

Explanans: The unbound exponential birth rate of a population of organisms $N$ at time $t^{0} \rightarrow$ Explanandum: determines the demographics of the population $N$ at time later time $t^{n}$.

It might seem that such a pattern is just as uninformative as our starting generalization of ecology (GenEc). However, we should recall that we are not required to evaluate this pattern and its model in isolation, but as a branch of the initial generalization. As such this model should be more contrastive then GenEc. The exponential growth pattern offers understanding only as an extension of the general assumptions of GenEc; by specifying only one of the factors (that of birth rate) it effectively negates environmental and inter-species factors. This creates a contrast with all the situations in which such factors have a noticeable effect on the population. Interestingly, even this simple expression of exponential growth has some domain of application. In microbiology exponential growth is used to describe microorganisms' growth until nutrient exhaustion (Slavov et al 2014). In epidemiology, it is employed as a starting point for explaining pandemic diseases (Chowell and Viboud 2016; Brauer and Castillo-Chavez 2012). Finally, in ecology, it is used for describing the behaviour of the prey when there are sufficient nutrients and an absence of predators (Arditi \& Ginzburg 2012).

Returning to our initial structure, we can extend the root pattern (GenEc), by introducing a relation with another species. This brings about the model:

$$
\text { (eq. 3) } \frac{\mathrm{dP}}{\mathrm{dt}}=\mathrm{Pf}_{\mathrm{p}}(\mathrm{P}, \mathrm{N}, \mathrm{E})
$$

This new equation stands on the same level of generality as our initial equation (1), because it simply introduces another population of organisms. Similarly, it can only be made more precise if the two populations are coupled. A straightforward coupling of equation (1) and (3) would be a case of predation, where $\mathrm{P}$ is a predator and $\mathrm{N}$ prey. This results in the pattern of basic predation: 
BasicPred:

Explanans: If a population P's growth depends on a resource provided by the member of a population $N \rightarrow$ Explanandum: then the harvesting of $N$ will hamper the growth of $N$ and benefit the growth of $P$.

In this case we can assume that the resources that $\mathrm{P}$ depends on are the members of $\mathrm{N}$, and that harvesting directly diminishes the population of $\mathrm{N}$ and benefits $\mathrm{P}$. Adding our previous notion of exponential growth of $\mathrm{N}$ and an extra assumption of the death rate of $\mathrm{P}$, leads us to the Lotka-Volterra equations (Volterra 1926):

$$
\begin{aligned}
& \frac{\mathrm{dN}}{\mathrm{dt}}=\mathrm{rN}-\mathrm{aNP} \\
& \frac{\mathrm{dP}}{\mathrm{dt}}=\mathrm{eaNP}-\mathrm{qP}
\end{aligned}
$$

Here $N$ and $P$ are the demographics of prey and predators, respectively; $r N$ and $q P$ designate the birth rate of the prey and the death rate of the predators, respectively; $e$ represents the efficiency of converting prey biomass to predator biomass; and $a$ the searching efficiency or the attack rate of predators. Of central importance for these equations are the so-called numerical and functional responses. The functional response is the intake rate of the predators as a function of the density of the prey. Here it is represented by the kill rate $a N P$. The numerical response is the change in predator density as a function of change in prey density; it is given in equation 3.2 by a function of the kill rate $a N P$ that depends only on the efficiency $e$ of the conversion of prey biomass to predator biomass.

This short overview of the Lotka-Volterra equations shows that they introduce quite a few new details into our hierarchical organization of ecological modelling. Although the equations remain highly idealized, they nevertheless serve as a generalization by which we can distinguish between the three possible population states of a coupled predator-prey populationecological balance, double extinction and the extinction of the predator. As such the specification of the variables of these models and addition of specific parameters can be used to describe (or zero in to) a specific population state or dynamics (Abrams \& Ginzburg 2000; Petkov 2019). If this state is representative of some targeted ecological populations, the model can also 
be used to derive specific explanations as to why such a state has been observed.

Without going into further details by describing more specific models, ones that have different functional responses, or take into account the carrying capacity of the environment or the satiation of the predators, we can organize the equations introduced so far into a structural hierarchy and investigate its properties. These ecological models can be given as the following preliminary tree:

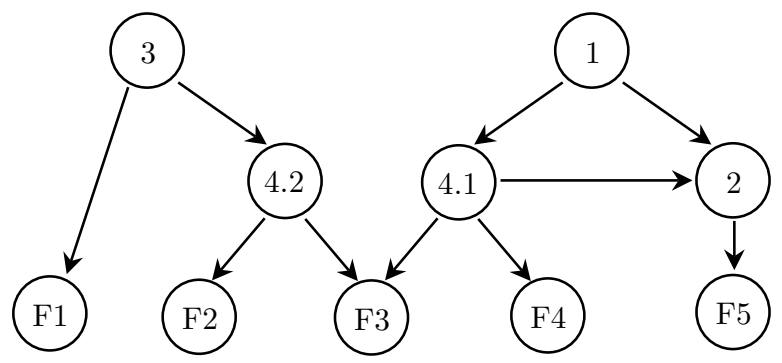

Fig.5

In this graph, the numbered nodes correspond to the equations (1 to 4.2) and the leaves (F1 to F5) can be assumed to represent specific explanations that can be derived from these models. Here, these specific explanations zero in not on singular facts but instead on the type of facts that they cover. As mentioned earlier, by adding more specific parameters we can use these models to describe and explain specific ecological facts. Although the resulting picture is significantly simplified, it nevertheless illustrates that F5 can stand for populations that can be explained by the exponential growth model (2.); F3 can represent facts related to an equilibrium between predator and prey; F4, facts related to population dynamics, in the absence of predators but considering limiting environmental factors; F2, a situation of prey extinction; and F1, a situation in which the other species is also coupled with resources other than the prey. It also shows that F1 is a type of population states that yet lack specific descriptions within our theory. Importantly the structure also designates that there is a relation between equations (4.1.) and (2.), as, in the absence of predators, the equation (4.1) collapses to the earlier equation of exponential growth (2.). (For 
further analysis on the relation between exponential growth models and the Lotka-Volterra model see Arditi and Ginzburg 2012).

We can appreciate that the emerging ecological theory of predator-prey dynamics is describable as a balanced tree and that it can be treated as an example of a unified explanatory nexus that also presents a complete explanatory structure, at least for the basic states of predator-prey populations - unlimited growth, ecological balance, double extinction and the extinction of the predator. As such, this hierarchy of models can serve as a good illustration of a theory that satisfies the ideas of optimal organization of inferential explanatory patterns in terms of their relations. Consequently, such a structure can be used to also describe the understanding of specific population states, as contrasted with other possible scenarios for population dynamics. For instance, the discipline of conservation biology requires such contrast, since it aims at maintaining and explaining biodiversity by defining the conditions under which an ecological system maintains balance, as opposed to various extinction scenarios.

\section{Conclusion}

Understanding of phenomena via an explaining theory requires both the right type of epistemic agents and the right kind of theory. As such understanding has two complementary dimensions - a subjective and an objective one. Whilst under the concept of "grasping" the analysis of the subjective dimension has undoubtedly shown that explanations cannot be considered in isolation, the analysis of the objective dimension has been mostly preoccupied only with uncovering the internal inferential relations within singular explanations. Criteria for evaluating the specific structure and organization of explanatory clusters or nexuses has received much less attention. The present study aimed at filling this gap.

As we saw the particular features of the explanatory inference and the position of the explanations within a theoretic hierarchy of explanations are equally important objective criteria for evaluating the resulting explanatory understanding. Moreover, the position of particular explanations within such structures can potentially amend some of these explanations' shortcomings as for instance if more general and less informative explanations 
instantiate more stringent ones. In this short essay, I represented these structures as directed graph trees and thus aimed at providing more robust criteria for evaluating the degree of understanding that such explanatory structures can offer in terms of completeness and balance.

The short exposition on ecological modeling serves as further evidence that such structural hierarchies are not a purely abstract idea and at least some fragments of scientific theories can be represented as such.

\section{Funding}

This work is supported by China's Major National Social Science Project—19ZDA041.

\section{References}

Abrams, Peter A. and Ginzburg, Lev R. 2000. "The Nature of Predation: Prey-Dependent, Ratio-Dependent or Neither?" Trends in Ecology and Evolution 15

(8): 337-41. https://doi.org/10.1016/S0169-5347(00)01908-X

Arditi, Roger and Ginzburg, Lev R. 2012. How Species Interact: Altering the Standard View on Trophic Ecology. New York: Oxford University Press.

Bartelborth, Thomas. 2002. "Explanatory Unification." Synthese 130 (1): 91-107. https://doi.org/10.1023/A:1013827209894

Baumberger, Christoph, Beisbart, Claus, and Brun Georg. 2017. "What is Understanding? An Overview of the Recent Debates in Epistemology and Philosophy of Science." In Explaining Understanding: New Perspectives from Epistemology and the Philosophy of Science, edited by Stephen R. Grimm, Christoph Baumberger, and Sabine Ammon, 1-34. New York: Routledge.

De Regt, Henk W. 2015. "Scientific Understanding: Truth or Dare?" Synthese 192 (12): 3781-797. https://doi.org/10.1007/s11229-014-0538-7

Dowe, Phil. 2000. Physical Causation. New York: Cambridge University Press.

Friedman, Michael. 1974. "Explanation and Scientific Understanding." Journal of Philosophy 71 (1): 5-19. https://doi.org/10.2307/2024924

Ginzburg, Lev R. and Colyvan, Mark. 2004. Ecological Orbits: How Planets Move and Populations Grow. New York: Oxford University Press.

Grimm, Stephen R. 2010. "The Goal of Explanation." Studies in the History and Philosophy of Science 41 (4): 337-44.

https://doi.org/10.1016/j.shpsa.2010.10.006

Gurova, Lilia. 2017. "On Some Non-Trivial Implication of the View that Good Explanations Increase Our Understanding of Explained Phenomena." Balkan Journal of Philosophy 9 (1): 45-52. https://doi.org/10.5840/bjp2017914 
Halonen, Ilpo and Hintikka, Jaakko. 1999. "Unification-It's Magnificent but is it Explanation?" Synthese 120 (1): 27-47. https://doi.org/10.1023/A:1005202403274

Hempel, Carl G. 1965. "Aspects of Scientific Explanation." In Aspects of Scientific Explanation and Other Essays in the Philosophy of Science, 331-96. New York: Free Press.

Holt, Robert D. 2011. "Natural Enemy-Victim Interactions: Do We Have a Unified Theory Yet?" In The Theory of Ecology, edited by Samuel M. Scheiner and Michael R. Willig, 125-61. Chicago: University of Chicago Press.

Kechris, Alexander S. 1994. Classical Descriptive Set Theory. Dordrecht: Springer. https://doi.org/10.1007/978-1-4612-4190-4

Khalifa, Kareem. 2017. Understanding, Explanation, and Scientific Knowledge. Cambridge, UK: Cambridge University Press.

Kitcher, Philip. 1989. "Explanatory Unification and the Causal Structure of the World." In Scientific Explanation, edited by Philip Kitcher and Wesley Salmon, 410-505. Minneapolis: University of Minnesota Press.

Knuth, Donald. 1998. The Art of Computer Programming. Volume 3: Sorting and Searching. Second Edition. Massachusetts: Addison-Wesley.

Kostić, Daniel. 2018. "Minimal Structure Explanations, Scientific Understanding and Explanatory Depth." Perspectives on Science 27 (1): 48-67.

https://doi.org/10.1162/posc_a_00299

Lange, Marc. 2016. Because Without Cause: Non-Causal Explanations in Science and Mathematics. New York: Oxford University Press.

Lewis, David. 2000. "Causation as Influence." Journal of Philosophy 97 (4): 18297. https://doi.org/10.2307/2678389

Morrison, Margaret. 2000. Unifying Scientific Theories. Cambridge: Cambridge University Press.

Newman, Mark. 2014. "EMU and Inference: What the Explanatory Model of Scientific Understanding Ignores." European Journal for Philosophy of Science 4 (1): 55-74.

Salmon, Wesley. 1998. Causality and Explanation. New York: Oxford University Press.

Schaffer, Jonathan. 2005. "Contrastive Causation." Philosophical Review 114 (3): 327-58. https://doi.org/10.1215/00318108-114-3-327

Petkov, Stefan. 2015. "Explanatory Unification and Conceptualization." Synthese 192 (12): 3695-717. https://doi.org/10.1007/s11229-015-0716-2

Petkov, Stefan. 2019. "Studying Controversies: Unification, Contradiction, Integration." Journal for General Philosophy of Science / Zeitschrift für Allgemeine Wissenschaftstheorie 50 (1): 103-28. https://doi.org/10.1007/s10838018-9431-2 
Reiss, Julian. 2013a. "Contextualising Causation Part I." Philosophy Compass 8 (11): 1066-75. https://doi.org/10.1111/phc3.12074

Reiss, Julian. 2013b. "Contextualising Causation Part II." Philosophy Compass 8 (11): 1076-90. https://doi.org/10.1111/phc3.12073

Van Fraassen, B. 1980. The Scientific Image. Oxford: Clarendon Press.

Volterra, Vito. 1926. "Fluctuations in the Abundance of a Species Considered Mathematically." Nature 118: 558-60. https://doi.org/10.1038/118558a0

Woodward, James. 2003. Making Things Happen. Oxford: Oxford University Press. https://doi.org/10.1111/j.1933-1592.2007.00012.x

Zager, Laura A. and Verghese, George C. 2008. "Graph Similarity Scoring and Matching." Applied Mathematics Letters 21 (1): 86-94.

https://doi.org/10.1016/j.aml.2007.01.006 